\title{
A note on Grzegorczyk's logic
}

\author{
Emil Jeřábek \\ Mathematical Institute, Czech Academy of Sciences \\ jerabek@math.cas.cz
}

December 1, 2003

\begin{abstract}
Grzegorczyk's modal logic ( Grz) corresponds to the class of upwards well-founded partially ordered Kripke frames, however all known proofs of this fact utilize some form of the Axiom of Choice; G. Boolos asked in [1], whether it is provable in plain $Z F$. We answer his question negatively: Grz corresponds (in $Z F$ ) to a class of frames, which does not provably coincide with upwards well-founded posets in $Z F$ alone.
\end{abstract}

Definition 1 Grzegorczyk's logic (Grz) [2] is a normal modal logic axiomatized by the schema

$$
\square(\square(\varphi \rightarrow \square \varphi) \rightarrow \varphi) \rightarrow \varphi .
$$

We denote by $\mathcal{K}_{1}$ the class of upwards well-founded posets, $\mathcal{K}_{3}$ the class of posets without any strictly increasing infinite chain, and $\mathcal{K}_{2}$ the class of posets $\langle W, \leq\rangle$ satisfying

$$
\forall X \subseteq W(X \neq \emptyset \rightarrow \exists x \in X \forall y \geq x \forall z \geq y(z \in X \rightarrow y \in X)) .
$$

Recall that the Principle of Dependent Choices $(D C)$ is the following weak version of the Axiom of Choice: let $R$ be a binary relation on a nonempty set $A$ such that $\forall x \in A \exists y \in A\langle x, y\rangle \in R$, then there is an infinite sequence $\left\{a_{n} ; n \in \omega\right\} \in A^{\omega}$ such that $\left\langle a_{n}, a_{n+1}\right\rangle \in R$ for every $n \in \omega$.

Lemma $2 Z F$ proves $\mathcal{K}_{1} \subseteq \mathcal{K}_{2} \subseteq \mathcal{K}_{3}$.

Proof: If $\langle W, \leq\rangle \in \mathcal{K}_{1}$, and $X \subseteq W$ nonempty, then any <-maximal element $x \in X$ witnesses that (1) holds, hence $\langle W, \leq\rangle \in \mathcal{K}_{2}$. Assume that there is $\langle W, \leq\rangle \in \mathcal{K}_{2} \backslash \mathcal{K}_{3}$. Fix an infinite increasing chain $x_{0}<x_{1}<x_{2}<\cdots$ in $W$, and put $X=\left\{x_{n} ; n\right.$ odd $\}$. Then for any $x \in X$ there are $z \geq y \geq x$ such that $z \in X$ and $y \notin X$, contradicting (1).

Proposition 3 (ZF $\vdash$ :) A frame $\mathbf{W}=\langle W, \leq\rangle$ is a model of Grz under all valuations if and only if $\mathbf{W} \in \mathcal{K}_{2}$. 
Proof: ("if") Let $\Vdash$ be a valuation in $\mathbf{W}, w \in W$, and $w \nVdash \varphi$. Define $X=$ $\{v ; w \leq v \& v \nVdash \varphi\}$, and let $x \in X$ be as in (1). If $y \geq x$, and $y \Vdash \varphi$, then $y \Vdash \square \varphi$ by $(1)$, hence $x \Vdash \square(\varphi \rightarrow \square \varphi)$, and $w \nVdash \square(\square(\varphi \rightarrow \square \varphi) \rightarrow \varphi)$.

("only if") It is well-known that Grz contains $S 4$, hence all Grz-frames are reflexive and transitive (i.e., preorderings). Assume that $X \subseteq W$ is a counterexample to (1), and put $w \Vdash p$ iff $w \notin X$, where $p$ is an atom. Let $x \in W$, and $x \Vdash \square(p \rightarrow \square p)$. This means $\forall y \geq x \forall z \geq y(y \notin X \rightarrow z \notin X)$, hence $x \notin X$ (by our assumption on $X)$, thus $x \Vdash p$. In other words, $\square(\square(p \rightarrow \square p) \rightarrow p)$ is valid in all nodes of $W$, however $p$ is not, because $X$ is nonempty. This contradicts $\mathbf{W} \Vdash G r z$.

Finally, notice that any preordering satisfying (1) is a partial ordering: taking $X=\{x\}$, (1) yields $x \geq y \geq x \rightarrow x=y$.

Lemma 4 The following are equivalent over $Z F$ :

(i) $D C$,

(ii) $\mathcal{K}_{1}=\mathcal{K}_{3}$,

(iii) $\mathcal{K}_{2}=\mathcal{K}_{3}$.

Proof: The implication $D C \rightarrow \mathcal{K}_{1}=\mathcal{K}_{3}$ follows directly from the definition, and $\mathcal{K}_{1}=\mathcal{K}_{3}$ implies $\mathcal{K}_{2}=\mathcal{K}_{3}$ by Lemma 2 , it remains to show $\mathcal{K}_{2}=\mathcal{K}_{3} \rightarrow D C$. Assume $\mathcal{K}_{3} \subseteq \mathcal{K}_{2}$, let $R \subseteq A^{2}$ be a relation without a maximal element, and let $a_{0} \in A$. Define $U$ as the set of all finite sequences $\left\langle a_{0}, \ldots, a_{n}\right\rangle \in A^{<\omega}$ such that $\left\langle a_{i}, a_{i+1}\right\rangle \in R$ for all $i<n$, ordered by inclusion (i.e., $s \leq t$ iff $t$ extends $s$ ). By taking $X=\{s \in U ; \operatorname{lh}(s)$ odd $\}$ we see that $U \notin \mathcal{K}_{2}$, hence (by assumption) $U \notin \mathcal{K}_{3}$. Consequently $U$ contains an infinite strictly increasing chain, and the union of such a chain is clearly an infinite sequence $\left\{a_{n} ; n<\omega\right\} \in A^{\omega}$ such that $\left\langle a_{i}, a_{i+1}\right\rangle \in R$ for all $i \in \omega$.

Proposition 5 There is a model of $Z F$, in which $\mathcal{K}_{1} \neq \mathcal{K}_{2} \neq \mathcal{K}_{3}$ (unless $Z F$ is inconsistent).

Proof: By Lemma 4, it suffices to find a model of $\mathcal{K}_{1} \neq \mathcal{K}_{2}$.

The following property holds in the Ordered Mostowski Model [7]: there is a dense linear ordering $\mathbf{W}=\langle W, \leq\rangle$ such that any subset of $W$ is a finite union of intervals. (Mostowski's permutation model is a model of $Z F A$, the set theory with atoms, but it is possible to transfer this result into $Z F$, using e.g. the Jech-Sochor Embedding Theorem [5], [6].) Clearly $\mathbf{W} \notin \mathcal{K}_{1}$, we claim that $\mathbf{W} \in \mathcal{K}_{2}$ : let $X$ be a nonempty subset of $W$, we may write $X$ as a disjoint union $X=I_{1} \cup \cdots \cup I_{n}$ of nonempty intervals (possibly degenerate) such that $I_{1}<\cdots<I_{n}$. Then any $x \in I_{n}$ witnesses (1).

Note: Halpern [3] has shown that the Boolean Prime Ideal Theorem (BPI) holds in Ordered Mostowski's Model (cf. also [4]), hence even $Z F+B P I$ doesn't prove $\mathcal{K}_{1}=\mathcal{K}_{2} \vee \mathcal{K}_{2}=\mathcal{K}_{3}$.

Corollary 6 It is relatively consistent with ZF that there is a Grz-frame which is not upwards well-founded. 


\section{References}

[1] G. Boolos, The Logic of Provability, Cambridge University Press 1993, p. 163

[2] A. Grzegorczyk, Some relational systems and the associated topological spaces, Fundamenta Mathematicae 60 (1967), pp. 223-231

[3] J. D. Halpern, The independence of the axiom of choice from the Boolean prime ideal theorem, Fundamenta Mathematicae 55 (1964), pp. 57-66

[4] T. Jech, The Axiom of Choice, North-Holland, Amsterdam 1973

[5] T. Jech, A. Sochor, On $\theta$-model of set theory, Bulletin de l'Académie Polonaise des Sciences 14 (1966), pp. 297-303

[6] T. Jech, A. Sochor, Applications of the $\Theta$-model, Bulletin de l'Académie Polonaise des Sciences 14 (1966), pp. 351-355

[7] A. Mostowski, Über die Unabhängigkeit des Wohlordnungssatzes vom Ordnungsprinzip, Fundamenta Mathematicae 32 (1939), pp. 201-252 